\title{
Do Tighter Restrictions on Density Retard Development?
}

\author{
Jyh-Bang Jou • Tan Lee
}

Published online: 27 February 2007

(C) Springer Science + Business Media, LLC 2007

\begin{abstract}
Williams (1991) builds a real-options model to investigate the timing and the scale decisions of property development. Williams asserts that tighter restrictions on density retard development. This article finds that there are some typos in Williams's article such that his assertion does not hold in general. In particular, his assertion will not hold as long as the density restrictions are not set too low relative to the density level that would be chosen by landowners in the absence of any regulation.
\end{abstract}

Keywords Development density $\cdot$ Real options

\section{Introduction}

The real-options model has been applied to decisions regarding many real assets such as those related to mining, energy and real estate, etc. Earlier studies on real options are typically concerned with the timing decision of investment (see, e.g., Dixit \& Pindyck, 1994). One exception is that of Williams (1991), which investigates both the timing and the scale decisions of property development. ${ }^{1}$ Williams assumes that the development costs are fully sunk and that undeveloped land can be developed once and for all. He then finds that, as uncertainty increases, a landowner will delay development, but will choose a larger scale of development once development proceeds. This contrasts with another type of investment (the socalled continuous investment) in which a firm can add capital infinitesimally because

\footnotetext{
${ }^{1}$ The other exceptions include Capozza and Li (1994), Childs, Riddiough, and Triantis (1996), and Williams (1997).
}

J.-B. Jou $(\bowtie)$

Graduate Institute of National Development, National Taiwan University,

No. 1 Roosevelt Rd. Sec. 4, Taipei 106 Taiwan, Republic of China

e-mail: jbjou@ntu.edu.tw

T. Lee

Department of International Business, Yuan Ze University,

No. 135 Yuan-Tung Rd., Chung-Li, Taoyuan 320 Taiwan, Republic of China

e-mail: tanlee@saturn.yzu.edu.tw 
greater uncertainty will then delay investment, or, equivalently, will reduce the firm's "desired" capital stock (see, e.g., Bar-Ilan \& Strange, 1999).

In Williams's framework, it is also possible to discuss how density restrictions affect the timing of property development. Williams finds that tighter density restrictions retard development. Given that density restrictions are typically more stringent as time goes by, it seems that Williams's finding conflicts with the overbuilding phenomenon that commonly occurs in the real estate market (Grenadier, 1996; Wang \& Zhou, 2000). The main purpose of this article is to point out that there are some typos in Williams's article. If these typos are corrected, then we will find that tighter density restrictions may either retard or enhance development. We also compare the corrected results with the literature of the certainty development models with density constraints (see Turnbull, 1991).

The remainder of this article is as follows. "Williams's Assumptions" section introduces the assumptions in Williams' model. "Choices of the Date and the Density of Development" section corrects the mistakes in Williams' results regarding choices of the date and the density of development. The last section compares the corrected results with those of the literature and also offers concluding remarks.

\section{Williams' Assumptions}

Consider a monopolized landowner in a real estate industry. As date $t=0$, the owner acquired his undeveloped property which is normalized at one unit. At anytime $t \geq 0$, the owner can develop his property once and for all at some feasible scale $q$, which is also equal to the density of development. The regulator imposes a constraint $\delta(>1)$ on the density of development $q$ such that $1 \leq q \leq \delta$. The development costs, which are fully irreversible, are given by

$$
C\left(x_{1}(t), q\right)=q^{\gamma} x_{1}(t)
$$

where the constant cost of scale $\gamma>1$ (which indicates that development at higher densities is more costly per unit of development), and $x_{1}(t)$ is a disturbance term which captures supply shocks. Suppose that $x_{2}(t)$ denotes the rent per unit of developed property. Both $x_{1}(t)$ and $x_{2}(t)$ follow geometric Brownian motions given by

$$
d x_{j}(t)=\mu_{j} x_{j}(t) d t+\sigma_{j} x_{j}(t) d Z_{j}(t),
$$

where $j=1,2$. Each variable $x_{j}(t)$ has a constant expected rate of growth $\mu_{j}$ and a constant variance of the growth rate $\sigma_{j}^{2}$. Each $\mathrm{d} Z_{j}(t)$ is an increment to a standard Wiener process, with $E\left\{\mathrm{~d} Z_{j}(t)\right\}=0$ and $E\left\{\mathrm{~d} Z_{j}(t)\right\}^{2}=\mathrm{d} t$. Furthermore, $E\left(\mathrm{~d} Z_{1}(t) \mathrm{d} Z_{2}(t)\right)=$ $\rho \sigma_{1} \sigma_{2} \mathrm{~d} t$, where $-1 \leq \rho \leq 1$.

Williams (1991) also adds the following assumptions in order to value both the undeveloped and developed properties: (1) the risk-less rate of interest $i$ is constant per unit of time; (2) the undeveloped property per unit has a constant positive return given by net cash inflow per unit of time $\beta x_{2}(t)$, where $\beta>0$; and (3) the stochastic evolution of both $x_{1}(t)$ and $x_{2}(t)$ can be replicated from portfolios of securities that are traded continuously without transaction costs in the perfectly competitive capital market. In this market there are two portfolios of traded securities, $j=1,2$, such that the returns on portfolio $j$ are perfectly locally correlated with the stochastic 
increments, $\mathrm{d} Z_{j}(t)$, in Eq. 2. For each portfolio, the excess mean return per unit of standard deviation equals some constant $\lambda_{j}$. In this case, the risk-adjusted expected growth rates, namely, $\nu_{j}=\mu_{j}-\lambda_{j} \sigma_{j}$, where $j=1,2$, are also constant.

The cash inflow for the landowner is derived by multiplying the rent per unit of developed property, $x_{2}(t)$, by the scale of developed property he owns, $q$, thus yielding $x_{2}(t) q$. Given that the landowner develops property once and for all and that the development costs are fully sunk, the value of developed property for the landowner is thus equal to the (time $t$ ) expected present value of the future cash flow given by

$$
P\left(x_{2}(t), q\right)=E_{t} \int_{t}^{\infty} e^{-i(\tau-t)} x_{2}(\tau) q \mathrm{~d} \tau=\pi q x_{2}(t),
$$

where $\pi=1 /\left(i-\nu_{2}\right)$. Here and in what follows, we assume that $i>\nu_{1}$ and $i>\nu_{2}$ so as to ensure that both the value of undeveloped land and the value of developed property are finite.

\section{Choices of the Date and the Density of Development}

Define $V\left(x_{1}(t), x_{2}(t)\right)$ as the value of undeveloped property. Given that the boundary condition for $V\left(x_{1}(t), x_{2}(t)\right)$ is satisfied, we can write

$$
V\left(x_{1}(t), x_{2}(t)\right)=A_{1} x_{2}(t)^{\eta} x_{1}(t)^{1-\eta}+\pi \beta x_{2}(t),
$$

where the first term on the right-hand side of Eq. 4 captures the option value from waiting, $A_{1}$ is a constant to be determined, and $\eta(>1)$ is the larger root of the quadratic equation given by

$$
\varphi(\theta)=-\frac{\sigma^{2}}{2} \theta(\theta-1)-\theta\left(v_{2}-v_{1}\right)+\left(i-v_{1}\right)=0
$$

where $\sigma^{2}=\sigma_{1}^{2}-2 \rho \sigma_{1} \sigma_{2}+\sigma_{2}^{2}$.

The landowner simultaneously chooses the timing and the scale of development. Suppose that $x_{1}^{*}$ and $x_{2}^{*}$ denote the respective values of $x_{1}(t)$ and $x_{2}(t)$ that trigger development, and that $q^{*}$ denotes the chosen density of development upon land that is developed. Define $y(t)=x_{2}(t) / x_{1}(t)$, where a higher value of $y(t)$ indicates that the state of nature is better because it comes from a larger value of $x_{2}(t)$ and/or a smaller value of $x_{1}(t)$, i.e., when demand for developed property is increased and/or the cost of developing land is reduced. Because the value function $V(\cdot)$ is linearly homogeneous in $x_{1}(t)$ and $x_{2}(t)$, we can also write $y(t)=x_{2}(t) / x_{1}(t), W(y(t))=V\left(x_{1}(t)\right.$, $\left.x_{2}(t)\right) / x_{1}(t)$, and $p(y(t), q)=P\left(x_{2}(t), q\right) / x_{1}(t)$, where

$$
\begin{gathered}
W(y(t))=A_{1} y(t)^{\eta}+\pi \beta y(t), \\
p(y(t), q)=\pi q y(t),
\end{gathered}
$$


Define $y^{*}\left(=x_{2}^{*} / x_{1}^{*}\right)$ as the optimal timing of development. The solution for $y^{*}, q^{*}$, and $A_{1}$ can be solved from the three conditions given by

$$
\begin{gathered}
W\left(y^{*}\right)=p\left(y^{*}, q^{*}\right)-q^{*} \gamma \\
\frac{\partial W\left(y^{*}\right)}{\partial y}=\frac{\partial p\left(y^{*}, q^{*}\right)}{\partial y}, \\
q^{*}=\arg \max _{\mathrm{q}}\left\{p\left(y^{*}, q\right)-q^{\gamma}: 1 \leq q \leq \delta\right\} .
\end{gathered}
$$

Equations 8 and 9 are the value-matching and the smooth-pasting condition, respectively. Define $Z\left(y^{*}, q^{*}\right)$ as the value of undeveloped property minus the net value of developing immediately, i.e., $W\left(y^{*}\right)-p\left(y^{*}, q^{*}\right)+q^{*}$, which is equal to zero by Eq. 8. Solving from Eqs. 8 and 9 yields

$$
Z\left(y^{*}, q^{*}\right)=\frac{\pi}{\psi} y^{*}\left(\beta-q^{*}\right)+q^{*} \gamma=0
$$

where $\psi=\eta /(\eta-1)>1$.

Equation 10 indicates that the chosen density, $q^{*}$, maximizes the net value of development. Define $X\left(y^{*}, q^{*}\right)$ as the marginal benefit from development minus the marginal cost of development, which is derived by setting the partial derivative of the terms on the right-hand side of Eq. 10 with respect to $q$ equal to zero. This yields

$$
X\left(y^{*}, q^{*}\right)=\pi y^{*}-\gamma q^{*}{ }^{*-1}=0
$$

Solving Eqs. 11 and 12 simultaneously and considering the constraint $1 \leq q^{*} \leq \delta$ yields the result as shown in Eq. $13:^{2}$

$$
\left\{\begin{array}{lcc}
\begin{array}{l}
\text { Constraint } \\
\psi
\end{array} & y^{*} & q^{*} \\
\frac{\delta-\beta}{\delta(1-\beta)} & 1 \\
\frac{\delta \psi}{\delta-\beta} \leq \gamma \leq \frac{\psi}{1-\beta} & \frac{\gamma}{\pi}\left(\frac{\beta \gamma}{\gamma-\psi}\right)^{\gamma-1} & \frac{\beta \gamma}{\gamma-\psi} \\
\gamma<\frac{\delta \psi}{\delta-\beta} & \frac{\psi \delta^{\gamma}}{\pi(\delta-\beta)} & \delta
\end{array}\right.
$$

\footnotetext{
${ }^{2}$ It is required that $\gamma>\psi$ for $q^{*}$ to be positive, which we assume here and in what follows. Springer
} 
However, Williams (1991) incorrectly adds " $\psi$ " in the numerators and denominators in Eq. 13, and writes the solution as

$$
\left\{\begin{array}{llc}
\begin{array}{l}
\text { Constraint } \\
\frac{\psi}{1-\beta \psi} \leq \gamma
\end{array} & y^{*} & q^{*} \\
\frac{\delta \psi}{\delta-\beta \psi} \leq \gamma \leq \frac{\psi}{1-\beta \psi} & \frac{\gamma}{\pi}\left(\frac{\beta \gamma \psi}{\gamma-\psi}\right)^{\gamma-1} & \frac{\beta \gamma \psi}{\gamma-\psi} \\
\gamma<\frac{\delta \psi}{\delta-\beta \psi} & \frac{\psi \delta^{\gamma}}{\pi(\delta-\beta \psi)} & \delta
\end{array}\right.
$$

With our corrected results shown by Eq. 13, we find that tighter density restrictions may retard or enhance development. By contrast, Williams asserts that tighter density restrictions retard development. ${ }^{3}$ In the following, we will explain the intuition behind our corrected results.

We will first depict the case in the absence of any regulation. The line $Z Z$ in Fig. 1 depicts the dependence of $y^{*}$ on $q^{*}$ defined in Eq. 11. This line has a positive slope, indicating that as the real estate industry becomes less densely developed ( $q^{*}$ is lower), the landowner will be more eager to develop his property ( $y^{*}$ is lower) because waiting will be less valuable. In addition, the line $X X$ in Fig. 1 depicts the dependence of $q^{*}$ on $y^{*}$ defined in Eq. 12. This line has a positive slope, indicating that the landowner will develop his property more densely at a better state. We also find that the slope for line $X X$ is larger than the slope for line $Z Z$ at the equilibrium point $\mathrm{A}$ in Fig. 1. In the absence of any regulation, Fig. 1 indicates that a developer will not develop until $y^{*}$ reaches a level equal to $\gamma(\beta \gamma /(\gamma-\psi))^{\gamma-1} / \beta$. At that instant, the landowner will develop land at a density equal to $\beta \gamma /(\gamma-\psi)$.

An imposed density ceiling that is greater than $\beta \gamma /(\gamma-\psi)$ will be irrelevant, while an imposed density ceiling that is lower than $\beta \gamma /(\gamma-\psi)$ will be binding. In Fig. 1, line $Z Z$ has a positive slope, which indicates that, given a lower density of development, a landowner will develop earlier. However, this does not necessarily imply that tighter restrictions on density will enhance development because the positive slope of line $Z Z$ appears only around the equilibrium point $A$, and does not necessarily appear in the region where density restrictions are binding. Differentiating $y^{*}$ with respect to $\delta$ around the binding region (i.e., $\delta<\beta \gamma /(\gamma-\psi)$ ) yields

$$
\begin{aligned}
\frac{\mathrm{d} y^{*}}{\mathrm{~d} \delta}=\frac{\psi \delta^{\gamma-1}}{\pi(\delta-\beta)^{2}}[\gamma(\delta-\beta)-\delta] & >0 \text { if } \frac{\beta \gamma}{\gamma-1}<\delta<\frac{\beta \gamma}{\gamma-\psi} . \\
& =0 \text { if } \delta=\frac{\beta \gamma}{\gamma-1} \\
& <0 \text { if } 1<\delta<\frac{\beta \gamma}{\gamma-1}
\end{aligned}
$$

\footnotetext{
${ }^{3}$ We also find the comparative-statics result of the timing of development ( $\left.y^{*}\right)$ with respect to the scale of cost convexity $(\gamma)$ is ambiguous. By contrast, Williams reaches a definite result (see Table 1 of Williams (1991), p. 197).
} 


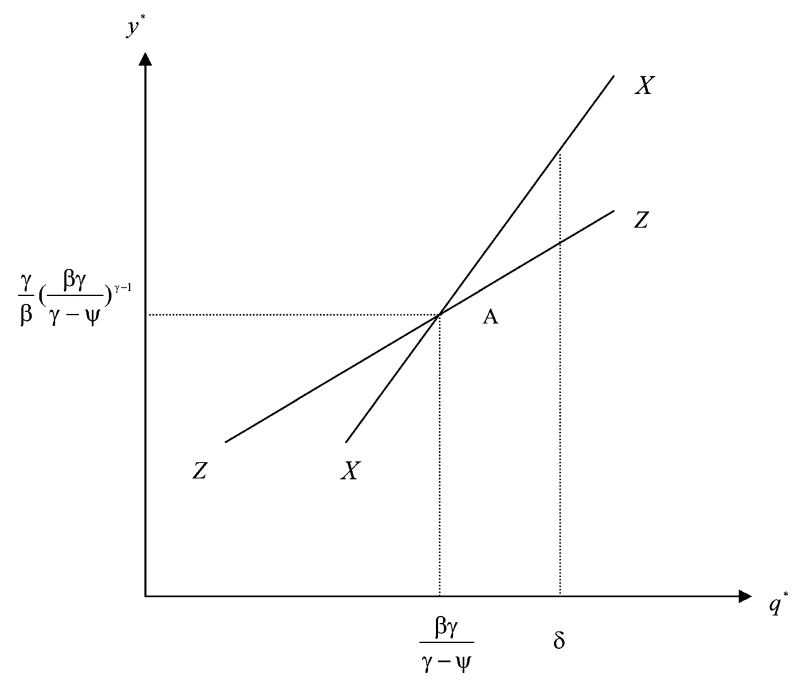

Fig. 1 In the absence of density ceiling control or the control is irrelevant

The result shown by Eq. 14 is depicted in Fig. 2. This Figure indicates that tighter restrictions will enhance development (by reducing $y^{*}$ ) if the imposed density ceiling is just decreased from $\beta \gamma /(\gamma-\psi)$, which is the optimal level of density chosen by a developer in the absence of any regulation. This is because the option value from waiting will then be reduced by a larger magnitude than the reduction in the net value of developing immediately. However, the situation changes if the imposed density ceiling is further reduced from a critical level equal to $\beta \gamma /(\gamma-1)$ (where $y^{*}$ will reach a minimum value equal to $\psi \gamma(\beta \gamma /(\gamma-1))^{\gamma-1} / \pi$. The option value from waiting will then be reduced by a smaller magnitude than the reduction in the net

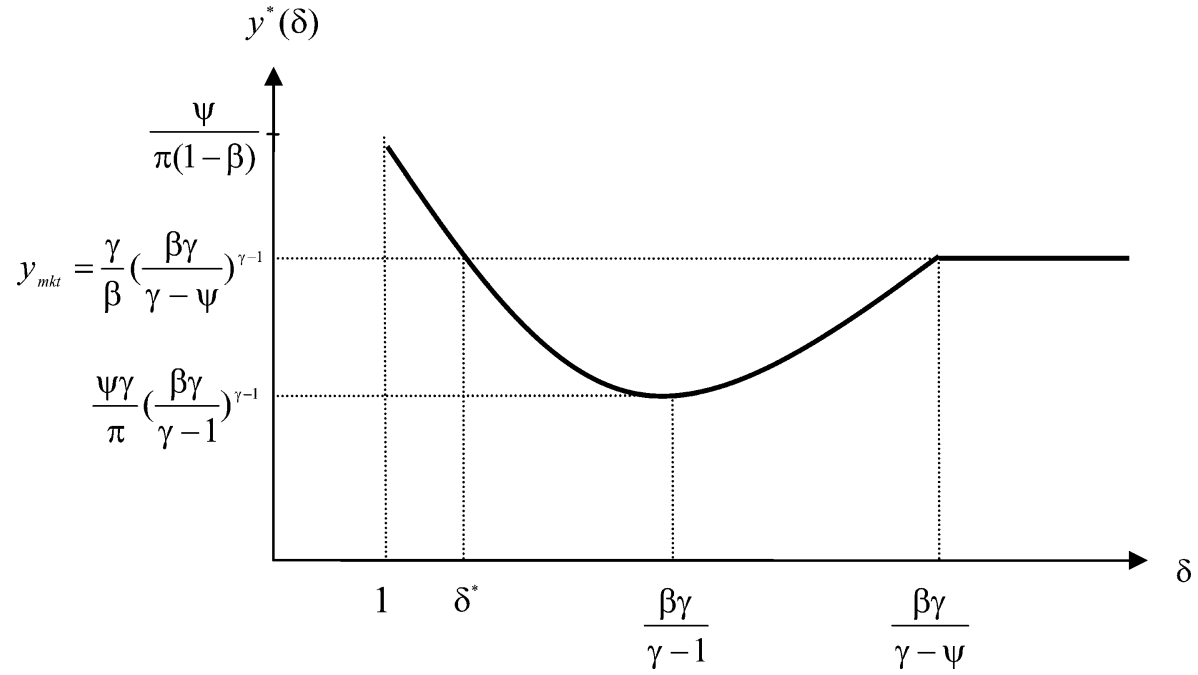

Fig. 2 The effect of density ceiling control 
value of developing immediately. Consequently, tighter restrictions on development density will retard development in this situation. ${ }^{4}$

\section{Conclusion}

Two articles including Titman (1985) and Turnbull (1991) also investigate the issue regarding how tighter restrictions affect property development. Williams assumes both that stochastic development cost has a specific functional form and that undeveloped land rents are proportional to developed land rents. By contrast, Titman neither imposes a specific functional form for stochastic development cost nor assigns an explicit role for undeveloped land rents. Titman finds that uncertainty about future developed rents will retard development. He further argues that the imposition of height restrictions on buildings may lead to an increase in the number of building units in an area because of the consequent decrease in uncertainty regarding the optimal height of the buildings. That is, he points out that tighter restrictions may enhance development.

Turnbull (1988) investigates residential development process using a dynamic model of an open city. Turnbull (1991) extends his earlier paper by allowing density ceiling constraints. Both papers assume that all households commute regularly from their residential site to the central business district. Absentee landowners construct perfectly durable houses and rent these houses to households. Landowners select the lot size of houses and development time $t$ to maximize profits per unit of land. The urban area can then be classified into three sites. Turnbull (1991) shows that binding density ceilings can exhibit different effects on the choice of the timing of development for these three sites.

The structural difference between the Williams's model used in this paper and the Turnbull's model (1991) is as follows: (1) the former assumes that the demanded density of development is increasing with the state of nature (The positive slope of $X X$ in Fig. 1 indicates this assumption), while the latter does not restrict the density growth to a single pattern; ${ }^{5}$ (2) the former allows for stochastic land rent and development cost, while the latter does not; and (3) the former assumes specific functional forms for rent evolution and development cost, while the latter does not. With this difference, Turnbull is able to depict the dynamic adjustment when the density ceiling control is binding. It is interesting to see that Turnbull shows that the effect of density ceiling control on the date of development depends on the type of sites (halting effects for site A and enhancing effects for sites B and C). By contrast, we find that the effect of density ceiling control exhibits an ambiguous effect on the choice of the development even for a single site, which resembles site $B$ in Turnbull's framework. Further study may consider the other two sites pointed out by

\footnotetext{
${ }^{4}$ Note that in Fig. 2, we assume that $\psi /\left(\pi(1-\beta)\right.$ ) (which is the $y^{*}$ corresponding to $\delta=1$ ) is larger than $\gamma(\beta \gamma /(\gamma-\psi))^{\gamma-1} / \beta$ (which is the $y^{*}$ corresponding to ). However, our results will still hold for the case where $\psi /(\pi(1-\beta))$ is smaller than or equal to . Specifically, Figure 2 applies to the case where the return to the undeveloped land $(\beta)$ is sufficiently large and the convexity of the development cost $(\gamma)$ is sufficiently small.

${ }^{5}$ In Turnbull's model, the demanded density of development is increasing over time for sites B and C, but is decreasing over time for site A.
} 
Turnbull (1991) through changing the functional forms regarding both the development costs and the value of developed property shown by Eqs. 1 and 3, respectively.

Despite the difference in the Williams-Titman-Turnbull approaches, they all yield the same conclusions for binding density restrictions (which hasten expected development time) as long as the density restrictions are not set too low relative to the market solution. ${ }^{6}$ We explain this as follows: Define $y^{*}(\delta)$ as the value read off the heavy curve in Fig. 2. Let $y_{m k t}$ denote the unconstrained value of the chosen date of property development. Let us also define $\delta^{*}$ as the $\delta$ where this heavy curve cuts the $y_{m k t}$ from above (i.e., $\delta^{*}$ is defined by $y^{*}\left(\delta^{*}\right)=y_{m k t}$ in Fig. 2). Now notice that $y^{*}(\delta)<y_{m k t}$ means expected timing speeds up (relative to the unregulated market) in response to the regulation, which is precisely the conclusions of Titman and Turnbull. The only outcome missing from both Titman and Turnbull is the possibility of $1<\delta<\delta^{*}$. However, when $\delta$ is so low it might force a landowner to foreclose development entirely. Consequently, once we correct Williams's (incorrect) claim, the corrected result will be reconcilable with the result of both Titman and Turnbull.

Acknowledgements We would like to thank the editor (James B. Kau), one anonymous reviewer, Ning Gong, and seminar participants at the Asian Finance Association 2005 Annual Conference. Financial support under Grant NSC 90-2416-H-155-027 from the National Science Council, Executive Yuan, R. O. C., is gratefully acknowledged.

\section{References}

Bar-Ilan, A., \& Strange, W. C. (1999). The timing and intensity of investment. Journal of Macroeconomics, 21(1), 57-77.

Capozza, D., \& Li, Y. (1994). The intensity and timing of investment: The case of land. American Economic Review, 84, 889-904.

Childs, P. D., Riddiough, T. J., \& Triantis, A. J. (1996). Mixed uses and the redevelopment option. Real Estate Economics, 24(3), 317-339.

Dixit, A. K., \& Pindyck, R. S. (1994). Investment under uncertainty. New Jersey: Princeton University Press.

Grenadier, S. R. (1996). The strategic exercise of options: Development cascades and overbuilding in real estate. Journal of Finance, 51(5), 1653-1679.

Titman, S. (1985). Urban land prices under uncertainty. American Economic Review, 75, 505-514.

Turnbull, G. K. (1988). Residential development in an open city. Regional Science and Urban Economics, 19, 307-320.

Turnbull, G. K. (1991). A comparative dynamic analysis of zoning in a growing city. Journal of Urban Economics, 29, 235-248.

Wang, K., \& Zhou, Y. (2000). Overbuilding: A game-theoretical approach. Real Estate Economics, 28(3), 493-522.

Williams, J. T. (1991). Real estate development as an option. Journal of Real Estate Finance and Economics, 4, 191-208.

Williams, J. T. (1997). Redevelopment of real assets. Real Estate Economics, 25(3), 387-407.

\footnotetext{
${ }^{6}$ We especially thank the reviewer for pointing out this.

Springer
} 